\title{
Níveis de potência muscular em atletas de futebol e futsal em diferentes categorias e posições
}

\author{
Levels of muscle power in soccer and futsal athletes of different \\ categories and positions
}

\author{
J.F. Silva, D. Detanico, L.T. Floriano, N. Dittrich, P.C. Nascimento, S.G. Santos, L.G.A. \\ Guglielmo
}

ARTIGO ORIGINAL | ORIGINAL ARTICLE

\begin{abstract}
Este estudo objetivou identificar a potência de membros inferiores em jogadores de futebol e futsal, comparando o counter movement jump (CMJ) entre modalidades, posições adotadas e categorias. Participaram 61 atletas de futebol das categorias Sub17, Sub20 e profissional e 28 de futsal das categorias Sub20 e profissional. Utilizou-se uma plataforma de força Quattro Jump do tipo piezoelétrica. Foi utilizada estatística descritiva, teste t de Student e análise de variância a $p<.05$. Não foi reportada diferença significativa no CMJ entre os atletas de futebol e futsal $(p=.85)$. Entre as posições adotadas pelos jogadores de futebol encontrou-se diferença significativa $(p=.02)$ apenas entre os centrais e os médios. Nos atletas de futsal não foi verificada diferença significativa entre as posições $(p=.69)$. Quando comparadas as categorias também não foram constatadas diferenças significativas, tanto no futebol $(p=.12)$, quanto no futsal $(p=.47)$. Pode-se concluir que os atletas de futebol e futsal parecem apresentar níveis semelhantes de potência muscular. Os centrais apresentaram maiores níveis de potência quando comparados aos médios, porém no futsal os atletas apresentaram níveis similares de potência. Independente da categoria, tanto os atletas de futebol quanto de futsal, apresentaram níveis semelhantes de potência muscular.
\end{abstract}

Palavras-chave: desempenho, futebol, futsal, potência muscular

This study aimed to identify the height obtained in the power of lower limb in soccer and futsal players, comparing the counter movement jump (CMJ) among the modalities, game positions and categories. Took part of the study 61 soccer athletes from under-17, under-20 and professional categories and 28 futsal athletes from the under-20 and professional categories. It was used a piezoelectric force platform Quattro jump. It was used descriptive statistics, student " $t$ " test and analysis of variance set at $p<.05$. No significant difference was reported in the CMJ between the soccer and futsal athletes $(p=.85)$. Among the positions adopted by soccer players it was found significant difference $(p=.02)$ only between those in defensive positions and those in the midfield. In the futsal athletes it was not reported significant difference between positions $(p=.69)$. Compared to other categories no significant differences were found, neither in soccer $(p=.12)$ nor in futsal $(p=$ .47). It can be concluded that the soccer and futsal athletes seems to present similar level of muscle power. Those in defensive positions presented higher levels of power when compared to those in the midfield, but in futsal the athletes presented similar power levels. Independent of the category, both soccer and futsal athletes presented similar level of muscle power.

Keywords: performance, soccer, futsal, muscle power

Submetido: 01.12.2009 | Aceite: 10.01.2011

Juliano Fernandes da Silva, Leandro Teixeira Floriano, Naiandra Dittrich, Paulo César Nascimento e Luiz Guilherme Antonacci Guglielmo. Laboratório de Esforço Físico, Programa de Pós-Graduação em Educação Física, Universidade Federal de Santa Catarina, Florianópolis, Santa Catarina, Brasil.

Daniele Detanico e Saray Giovana dos Santos. Laboratório de Biomecânica, Programa de Pós-Graduação em Educação Física, Universidade Federal de Santa Catarina, Florianópolis, Santa Catarina, Brasil.

Endereço para correspondência: Daniele Detanico, Laboratório de Biomecânica, Centro de Desportos UFSC, Campus Universitário da Trindade, CEP: 88040-900, Florianópolis - SC, Brasil

E-mail: danieledetanico@gmail.com 
O futebol e o futsal de alto nível são modalidades intermitentes de elevada intensidade caracterizadas por períodos curtos de recuperação e em que acções rápidas e intensas assumem particular importância durante o jogo (Bangsbo, 1994; Buchheit et al., 2009; Castagna, D’Ottavio, Granda-Vera, \& Alvarez, 2008; Di Salvo et al., 2007; Reilly \& Thomas, 1976).

A planificação do treinamento nesses esportes tem um cariz complexo tendo em vista as diferentes dimensões envolvidas, como o treinamento técnico, tático, psicológico e físico, necessitando, deste modo que estes aspectos sejam abordados de forma integrada (Castagna et al., 2008).

Com relação à condição física, estudos recentes têm destacado os componentes aeróbio (potência e capacidade) e anaeróbio como principais aspetos associados à performance de atletas de futebol (Helgerud, Engen, Wis-loff, \& Hoff, 2001; Impellizzeri, Rampinini, \& Marcora, 2005; Spencer, Bishop, Dawson, \& Goodman, 2005). Embora o futebol e o futsal apresentem predominância aeróbia, a aptidão anaeróbia demonstrada em acções de potência muscular, está relacionada com atividades decisivas das partidas como sprints, saltos, chutes e tackles (Abrantes, Maçãs, \& Sampaio, 2004; Soares \& Tourinho Filho, 2006; Wragg, Maxwell, \& Doust, 2000).

No que diz respeito aos movimentos característicos num jogo de futebol, tem sido observado que um sprint ocorre em média a cada 90s (Reilly \& Thomas, 1976) com durações entre 2 s e 4 s (Spencer et al., 2005). Além disso, os sprints constituem 1 a $11 \%$ da distância total percorrida na partida, correspondendo de 0.5 a $3 \%$ do tempo do jogo (Hoff \& Helgerud, 2004). Por outro lado, no futsal tem sido verificado que $13.7 \%$ dos deslocamentos são realizados em alta intensidade $(18.1-25.0 \mathrm{~km} / \mathrm{h})$ e $8.9 \%$ na forma de sprints (acima de $25 \mathrm{~km} / \mathrm{h}$ ) (Barbero-Alvarez, Soto, Barbero-Alvarez \& Granda-Vera, 2008), correspondendo a tarefas de solicitação neuromuscular durante toda a partida. Isto demonstra que apesar de serem de modalidades diferentes, possuem caracterís- ticas mecânicas e fisiológicas comuns (Matos et al., 2008).

Em estudo prévio, Gorostiaga et al. (2009) verificaram que atletas profissionais de futsal obtiveram desempenho inferior no counter movement jump (CMJ) e nos tempos de sprints de 5 e $15 \mathrm{~m}$ quando comparados a futebolistas. Embora as razões para estas diferenças serem desconhecidas, podem existir distintas origens como as demandas físicas durante jogos (número de sprints, distância dos sprints, distância percorrida total e número de saltos), quantidade e tipo de treinamentos. Por outro lado, ainda que a distância de $15 \mathrm{~m}$ em sprint se apresente no futsal em situações de contraataque e por isso tendencialmente ocorrendo com menos frequência, e no futebol como sendo a distância média percorrida em alta intensidade (Edwards, Macfadyen, \& Clark, 2003), em ambas as modalidades esta se correlacionou com o CMJ (Gorostiaga et al., 2009). Isso sugere que o desempenho em exercícios de elevada intensidade é dependente do nível de potência muscular, ou seja, da capacidade de recrutamento neural, do aproveitamento do ciclo alongamento-encurtamento (CAE) e da taxa de liberação de energia por meio da via metabólica anaeróbia (Komi, 2000).

Outros estudos têm demonstrado associação entre acções anaeróbias e níveis de potência muscular em atletas de futebol profissionais. Wisloff, Castagna, Helgerud, Jones e Hoff (2004) reportaram correlação significativa entre sprints de 10 e $30 \mathrm{~m}$ e o squat jump - SJ ( $r$ $=.72, r=.60, p<.01$, respetivamente). Além disso, sprints em distâncias de $5 \mathrm{~m}$ têm sido associados à potência mensurada no $\mathrm{SJ}(r=$ $-.64, p<.01$ ) (Sleivert \& Taingahue, 2004), indicando que distâncias curtas requerem elevada capacidade de recrutamento neural. Tais aspectos estão relacionados com o desenvolvimento da capacidade de aceleração, aptidão essencial em atletas de futebol e futsal, por exemplo em acções ofensivas de desequilíbrio (Silvestre, West, Maresh, \& Kraemer, 2006).

Os parâmetros neuromusculares podem apresentar diferenças entre atletas que actuam 
em diferentes posições, conforme verificado por Wisloff, Helgerud e Hoff (1998), ao encontrarem que os defensores e os avançados apresentaram valores superiores no desempenho do CMJ, quando comparado aos futebolistas que atuavam no meio de campo. Isso se deve provavelmente ao fato de que atletas destas posições (avançados e defensores) estão permanentemente e potencialmente submetidos a um maior esforço muscular de potência, em relação aos médios.

Em adição, atletas de diferentes categorias também podem apresentar níveis de potência muscular diferenciados, tendo em vista que o potencial elástico do músculo esquelético é uma propriedade que pode ser melhorada através dos anos de treinamento (Bosco, 1999). Seabra, Maia e Garganta (2001) compararam alguns índices neuromusculares entre futebolistas e não-futebolistas de três faixas etárias (infantil: 10-12 anos, iniciado: 13-14 anos e juvenil: 15-16 anos) e reportaram diferença significativa em saltos verticais consecutivos (15 s) entre as categorias iniciado e juvenil. Tais resultados confirmam a hipótese que indivíduos mais avançados no processo de desenvolvimento são capazes de produzir resultados superiores em tarefas que envolvam capacidade de força ou de potência, como por exemplo, saltos, chutes e sprints.

Por outro lado, parece existir uma lacuna na literatura que compare índices neuromusculares entre categorias de base (escalões mais elevados) e a profissional, tanto no futebol quanto no futsal, assim como entre diferentes posições. Em função disso, os objetivos deste estudo foram comparar: (i) a potência de membros inferiores entre jogadores de futebol das categorias sub17, sub20 e profissional e entre atletas de futsal das categorias sub20 e profissional; (ii) entre jogadores que actuam em diferentes posições no futebol e no futsal; e (iii) entre atletas profissionais de futebol e futsal. Foram formuladas as seguintes hipóteses: (i) existe diferença nos níveis de potência entre jogadores de futebol e futsal; (ii) os jogadores da categoria adulta, tanto de futsal quanto de futebol, possuem maiores níveis de potência; e (iii) existe diferença nos níveis de potência em jogadores que atuam em diferentes posições.

\section{Amostra}

\section{MÉTODO}

Participaram, deste estudo descritivo, 61 atletas de futebol das categorias sub17 (16-17 anos), sub20 (18-20 anos) e profissional e 28 atletas de futsal das categorias sub20 (18-20 anos) e profissional. As características antropométricas dos atletas estão apresentadas na Tabela 1.

Tabela 1

Características antropométricas dos atletas de futebol e futsal

\begin{tabular}{lcc}
\hline \multicolumn{1}{c}{ Categorias $(n)$} & $\begin{array}{c}\text { Massa } \\
\text { corporal }(\mathrm{kg})\end{array}$ & $\begin{array}{c}\text { Estatura } \\
(\mathrm{cm})\end{array}$ \\
\hline Futebol & & \\
Sub17 (22) & $69.2 \pm 6.3$ & $176.5 \pm 6.8$ \\
Sub20 (20) & $75.4 \pm 8.4$ & $178.5 \pm 8.1$ \\
$\quad$ Profissional (19) & $79.7 \pm 10.1$ & $179.9 \pm 6.1$ \\
Futsal & & \\
$\quad$ Sub20 (14) & $69.6 \pm 5.4$ & $173.4 \pm 4.0$ \\
Profissional (14) & $76.9 \pm 8.7$ & $177.5 \pm 6.6$ \\
\hline
\end{tabular}

Os atletas de futebol profissionais são considerados de elite, pois participavam do Campeonato Brasileiro da $1^{\text {a }}$ divisão. Os atletas de futsal também são de elite, pois foram classificados entre as seis primeiras equipes da Liga Brasileira de Futsal. Para fins de análise, os atletas foram subdivididos nas seguintes posições para o futebol: guarda-redes, centrais, laterais, volantes, médios e avançados; para futsal: guarda-redes, centrais, pivôs e alas.

O presente estudo foi aprovado pelo Comitê de Ética em Pesquisas com Seres Humanos da UFSC, sob o processo 073/2007. Todos os atletas assinaram o Termo de Consentimento Livre e Esclarecido.

\section{Instrumentos e Procedimentos}

Avaliação antropométrica

Para as medidas de massa corporal e esta 
tura utilizou-se uma balança da marca Plena ${ }^{\circledR}$, calibrada em miligramas e um estadiômetro calibrado em centímetros.

\section{Avaliação da potência muscular}

Para a obtenção do CMJ utilizou-se uma plataforma de força Quattro Jump (KISTLER ${ }^{\circledR}$, modelo 9290AD) do tipo piezoelétrica, que faz medidas da força vertical. As informações adquiridas foram transmitidas via cabo a um computador na frequência de $500 \mathrm{~Hz}$.

\section{Recolha de dados}

As avaliações foram realizadas no Laboratório de Biomecânica da UFSC, durante o período de pré-temporada dos atletas de futebol e futsal na época 2008/ 2009.

Os atletas compareceram adequadamente vestidos para a realização da avaliação e foram informados sobre os procedimentos da mesma. Inicialmente, foi realizada avaliação antropométrica (massa corporal e estatura); em seguida os atletas realizaram um aquecimento prévio, constituído por alongamentos livres e pequenos saltos, e executaram o salto CMJ sobre a plataforma de força.

Para a execução do CMJ, os atletas partiram de uma posição em pé, com as mãos na cintura e executaram um contramovimento flexionando os joelhos até próximo a $90^{\circ}$ (Bosco, 1999). Foram realizadas três execuções para o CMJ, sendo considerada para a análise a média das mesmas. Utilizou-se a altura do salto como indicador de performance.

\section{Análise Estatística}

Utilizou-se estatística descritiva (média e desvio-padrão) para a apresentação dos dados, teste de Kolmogorov-Smirnov para verificar a normalidade, teste $t$ de Student para dados independentes para a comparação entre atletas de futebol e futsal, e análise de variância (ANOVA one way) seguida pelo teste de Tukey para a comparação entre as categorias e entre as posições adotadas pelos atletas. Foi adotado um $p<.05$.

\section{RESULTADOS}

Os atletas profissionais de futebol apresentaram valores médios de CMJ de $44.2 \pm 3.5 \mathrm{~cm}$ e os atletas de futsal de $43.8 \pm 6.8 \mathrm{~cm}$, não sendo observada diferença significativa ( $p=$ .85 ) entre ambos (ver Figura 1).

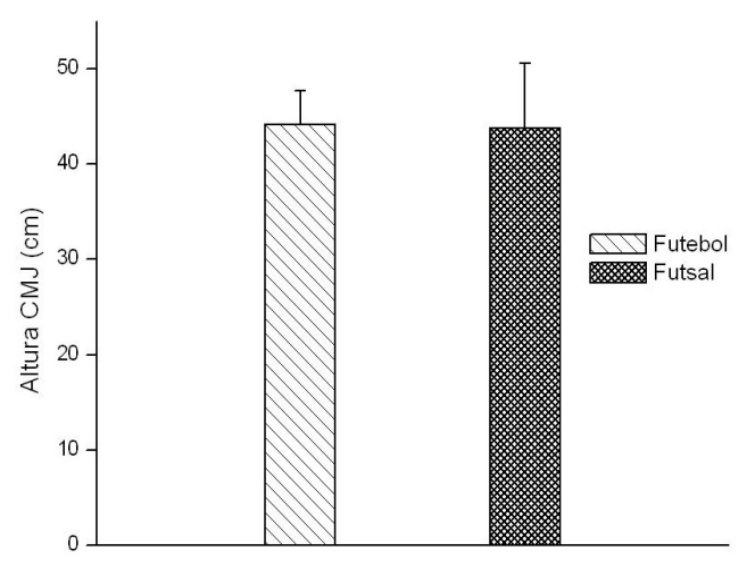

Figura 1. Comparação do CMJ entre os atletas profissionais de futebol e futsal

Com relação à análise das posições dos atletas, pode-se verificar que os valores médios apresentados pelos jogadores de futebol foram: médios $-41.4 \pm 2.5 \mathrm{~cm}$; laterais $-41.7 \pm 2.4$ $\mathrm{cm}$; avançados - $42.1 \pm 3.7 \mathrm{~cm}$; volantes $43.4 \pm 3.4 \mathrm{~cm}$; guarda-redes $-44.8 \pm 2.0 \mathrm{~cm} \mathrm{e}$ centrais $-45.8 \pm 4.3 \mathrm{~cm}$. Conforme a Figura 2 , apenas os centrais apresentaram diferença significativa $(p=.02)$ em relação aos médios.

Por sua vez, os atletas de futsal reportaram os seguintes valores do CMJ: pivôs $-41.5 \pm$ $4.8 \mathrm{~cm}$; alas $-42.5 \pm 3.8 \mathrm{~cm}$; centrais -45.1 $\pm 5.7 \mathrm{~cm}$ e guarda-redes $-46.6 \pm 7.9 \mathrm{~cm}$. Observando a Figura 3, verifica-se que não houve diferença significativa $(p=.69)$ entre os atletas de futsal das diferentes posições.

Quanto à análise por categorias, os futebolistas apresentaram valores médios do CMJ de $42.0 \pm 2.7 \mathrm{~cm}$ para a categoria sub17, $42.8 \pm$ $3.7 \mathrm{~cm}$ para a sub20 e $44.2 \pm 3.6 \mathrm{~cm}$ para a profissional. Os atletas de futsal reportaram valores médios de $43.7 \pm 4.1 \mathrm{~cm}$ para a categoria sub20 e $43.8 \pm 6.8 \mathrm{~cm}$ para os profissionais. 


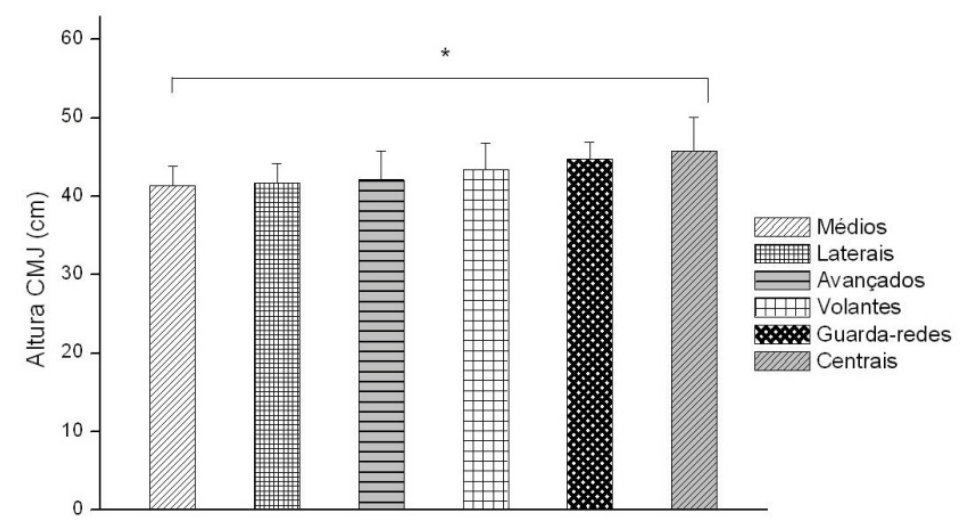

Figura 2. Comparação do CMJ entre os atletas de futebol de diferentes posições

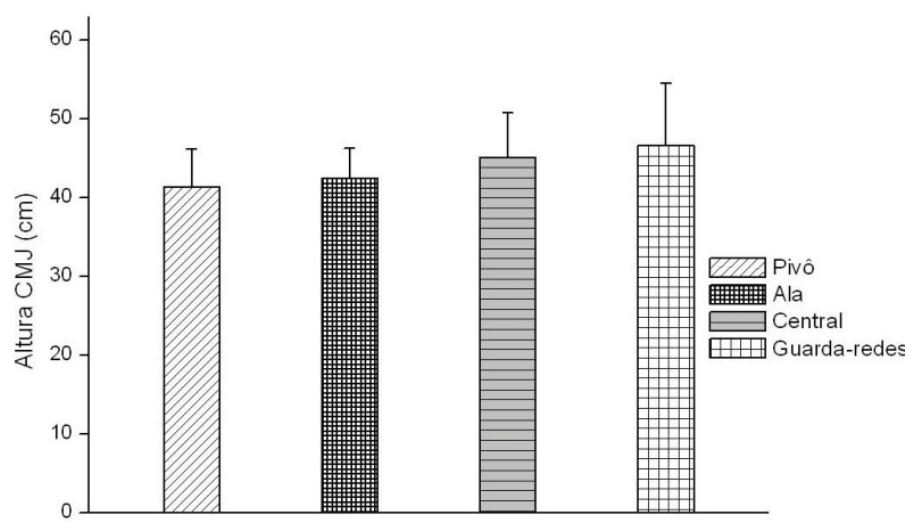

Figura 3. Comparação do CMJ entre os atletas de futsal de diferentes posições

De acordo com as comparações entre as categorias apresentadas nas Figuras 4 e 5, não foi constatada diferença significativa no desempenho do CMJ nas categorias analisadas, tanto no futebol $(p=.12)$ quanto no futsal $(p=.47)$.

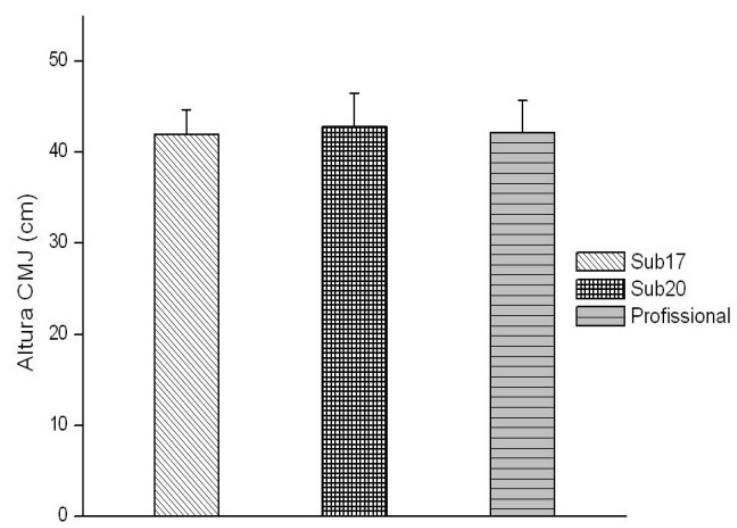

Figura 4. Comparação do CMJ entre os atletas de futebol das categorias Sub17, Sub20 e profissional

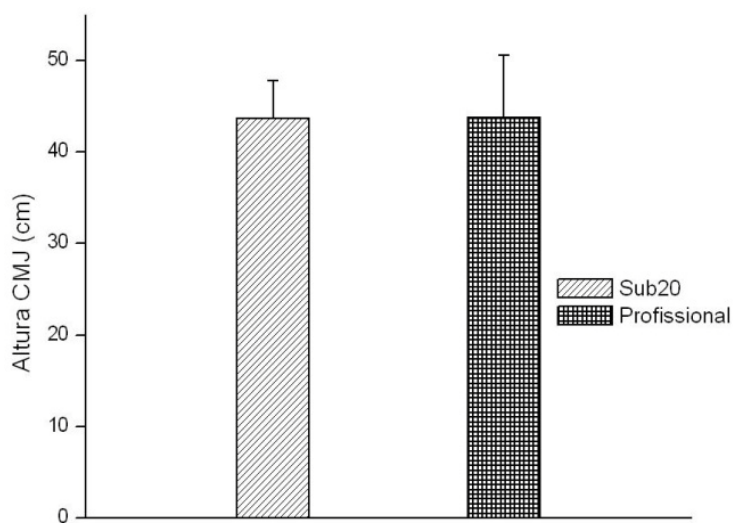

Figura 5. Comparação CMJ entre os atletas de futsal das categorias Sub20 e profissional

\section{DISCUSSÃO}

Um importante resultado do presente estudo foi que os atletas profissionais de elite de futsal e futebol não apresentaram diferenças significativas na altura atingida no CMJ (ver Figura 1). Apesar de serem modalidades com 
especificidade distinta em relação à distância percorrida, intensidade das corridas e espaço de jogo, têm movimentos similares do ponto de vista da potência muscular (Gorostiaga et al., 2009). Deste modo, podemos referir que, do ponto de vista da potência muscular, os atletas apresentam capacidades similares nas respostas às ações decisivas nos jogos, como por exemplo, os chutes, saltos, mudanças de sentido e direção.

Um fator que poderia ser considerado é a sensibilidade do salto CMJ para avaliar aspetos específicos da capacidade neuromuscular de atletas de futebol e futsal. Apesar de considerarmos a validade do CMJ para avaliar a capacidade neuromuscular (Markovic, Dizdar, Jukic \& Cardinale, 2004), é curioso o fato de atletas de futsal não apresentarem diferenças significativas nos índices neuromusculares em relação aos futebolistas. Em função do espaço no qual a modalidade é disputada, os atletas de futsal realizam atividades de alta intensidade relativamente mais curtas que atletas de futebol (Castagna et al., 2008; Hoff \& Helgerud, 2004), mas isto não foi suficiente para diferenciar os atletas de ambas as modalidades no CMJ, possivelmente pelo fato das ações decisivas dos futebolistas também ocorrerem em espaços curtos.

Além disso, poderia ser esperado que futebolistas apresentassem uma menor capacidade neuromuscular para realizar atividades explosivas que atletas de futsal, já que no futsal os momentos de atividades explosivas são tendencialmente mais constantes que no futebol (Castagna et al., 2008). Isto poderia sugerir que em avaliações mais específicas como sprints de 5 ou $15 \mathrm{~m}$, os atletas de futsal poderiam apresentar melhor desempenho. Contudo, Gorostiaga et al. (2009) demonstraram o contrário, ou seja, futebolistas também tendem a apresentar melhor desempenho em tais sprints, devido a maior produção de força concêntrica. No presente estudo, por não terem sido encontradas diferenças significativas no desempenho no CMJ entre jogadores de futebol e futsal sugere-se que o padrão de ativação neural e/ou a força de tensão por grupo muscular nas ações concêntricas de extensão do joelho não sejam muito díspares entre os atletas das duas modalidades.

Outro achado do presente estudo foi a diferença significativa encontrada entre os centrais e os médios no CMJ (Figura 2). Estes resultados corroboram os achados de Wisloff et al. (1998) que também reportaram ter encontrado diferenças significativas entre defensores e médios. Segundo os mesmos autores, este fato poderá justificar-se pelo fato de os centrais realizarem um maior número de tarefas de elevada intensidade, como saltos, sprints em distâncias curtas e desarmes.

Entre as demais posições no presente estudo não houve diferença significativa no CMJ. Barros et al. (2007) analisaram a distância percorrida durante uma partida de futebol entre as diferentes posições (defensores, alas, volantes, médios e avançados) em cinco velocidades [V1: caminhada e trote $(0-11 \mathrm{~km} / \mathrm{h})$; V2: corrida lenta (11-14 km/h); V3: corrida moderada (14-19 km/h); V4: alta velocidade (19-23 $\mathrm{km} / \mathrm{h}$ ); V5: sprinting ( $\geq 23 \mathrm{~km} / \mathrm{h}$ )]. Foi constatado não haver diferenças significativas na distância percorrida entre as diferentes posições, destacando-se que nas velocidades mais elevadas (V4 e V5), onde os atletas devem apresentar maiores esforços de potência, não houve diferença na distância percorrida. Este facto pode sugerir que atletas de diferentes posições devam possuir níveis de potência muscular similares, embora as tarefas desempenhadas no jogo sejam distintas, divergindo dos achados deste estudo. No entanto, deve-se ressaltar que na investigação supracitada não foram mensurados parâmetros de força muscular, apenas estimativas pela velocidade e distância percorrida, limitando possíveis afirmações.

Nos atletas de futsal também não foram encontradas diferenças significativas entre as posições adotadas (Figura 3); além disso, também não se encontraram estudos na literatura consultada que verificassem a distância percorrida por atletas de futsal de diferentes posições em elevada intensidade. O fato de não terem 
sido encontradas diferenças significativas no CMJ de atletas de futsal pode ser atribuído aos tipos de movimentos realizados, que compreendem acelerações e desacelerações em espaços curtos para acções de ataque ou defesa independente da posição em quadra. Por outro lado, muitas vezes as posições são definidas pela necessidade do time em um momento específico ou pelas características dos jogadores na quadra em determinado momento, podendo não haver uma especialização funcional tão acentuada como no futebol (BarberoAlvarez et al., 2008).

Outro aspeto observado neste estudo foi que jogadores de diferentes categorias de futebol e de futsal não apresentaram diferenças significativas no CMJ (Figuras 4 e 5). Analisando níveis de potência muscular em futebolistas de categorias de base, Seabra et al. (2001) reportaram diferença significativa entre futebolistas e não-futebolistas iniciados e juvenis apenas em saltos verticais consecutivos (15 s), não encontrando diferença na altura do SJ e CMJ; porém, este estudo fez a comparação apenas entre futebolistas e não-futebolistas, não apontando se existem diferenças entre as categorias de atletas de futebol.

Os resultados do presente estudo divergiram do sugerido na literatura, pois segundo Bosco (1999), o potencial elástico do músculoesquelético é uma propriedade que pode ser melhorada através dos anos de treinamento. Contudo, é importante ressaltar que no Brasil os atletas iniciam a sua rotina de treinamentos cada vez mais cedo, devido à possibilidade de integrar a categoria profissional a partir dos 16 anos. Isso permite que estes atletas de categorias de base desenvolvam precocemente as capacidades de força e potência muscular. Além disso, a produção de força e de potência é dependente de fatores genéticos, como o tipo de fibra muscular, pois um maior percentual de fibras rápidas é capaz de produzir elevados gradientes de força no início da contração (Bosco \& Komi, 1979), e consequentemente, melhor desempenho em saltos verticais.
Neste estudo foi analisado o CMJ como indicador marcador neuromuscular, tendo em vista que é o melhor indicador de potência de membros inferiores (Bosco, 1999). No entanto, talvez outros índices, como sprints de 5, 10 e $15 \mathrm{~m}$ possam apresentar diferenças entre os atletas de distintas faixas etárias e posições. Além disso, devido aos aspetos de desenvolvimento orgânico e níveis de treinamento, sugere-se que a capacidade de sustentar altos níveis de potência durante uma partida deve ser mais elevada nos atletas profissionais (Villar \& Denadai, 2001), tanto no futebol quanto no futsal.

\section{CONCLUSÕES}

Pode-se concluir que os atletas de futebol e futsal analisados apresentaram níveis semelhantes de potência de membros inferiores. Em relação às posições adotadas pelos atletas de futebol, apenas os centrais apresentaram maiores níveis de potência quando comparados aos médios. No futsal não existiram diferenças entre as posições ocupadas nos níveis de potência muscular avaliados. Por fim, independente da categoria (sub17, sub20 ou profissional), tanto os atletas de futebol quanto de futsal, apresentaram níveis semelhantes de potência muscular.

É importante ressaltar que embora os níveis de potência, em sua maioria, tenham sido similares nos atletas analisados, sugerem-se mais estudos que avaliem a potência muscular utilizando outras metodologias, como saltos unilaterais ou a utilização de aparelhos isocinéticos.

\section{Agradecimentos:}

Os autores gostariam de agradecer os integrantes do LAEF e BIOMEC que auxiliaram nas coletas de dados.

Conflito de Interesses:

Nada a declarar.

Financiamento:

Nada a declarar. 
Potência muscular em atletas de futebol e futsal | $\mathbf{2 1}$

\section{REFERÊNCIAS}

Abrantes, C., Maçãs, V., \& Sampaio, J. (2004). Variation in football players' sprint test performance across different ages and levels of competition. Journal of Sports Science and Medicine, 3(YISI1), 44-49.

Bangsbo, J. (1994). Fitness training in football: $A$ scientific approach. Baegsvard: Storm.

Barbero-Alvarez, J. C., Soto, V. M., Barbero-Alvarez, V., \& Granda-Vera, J. (2008). Match analysis and heart rate of futsal players during competition. Journal of Sports Sciences, 26(1), 63-73.

Barros, R. M., Misuta, M. S., Menezes, R. P., Figueroa, P. J., Moura, F. A., Cunha, A. S., ... Leite, N. J. (2007). Analysis of the distances covered by first division Brazilian soccer players obtained with an automatic tracking method. Journal of Sports Science and Medicine, 6(2), 233-242.

Bosco, C. (1999). Strength assessment with the Bosco's test. Rome: Italian Society of Sport Science.

Bosco, C., \& Komi, P. V. (1979). Mechanical characteristics and fiber composition of human leg extensor muscle. European Journal of Applied Physiology, 41 (4), 275-284.

Buchheit, M., Lepretre, P., Behaegel, P., Millet, G., Cuvelier, G., \& Ahmaidi, S. (2009). Cardiorespiratory responses during running and sport-specific exercises in handball players. Journal of Science and Medicine in Sport, 12(3), 399-405. doi: 10.1016/j.jsams.2007.11.00

Castagna, C., D’Ottavio, S., Granda-Vera, J., \& Alvarez, B. (2008). Match demands of professional futsal: A case study. Journal of Science and Medicine in Sport, 12(3), 491-494. doi: 10.1016/ j.jsams.2008.02.00

Di Salvo, V., Baron, R., Tschan, H., Calderon Montero, F.J., Bachl, N., \& Pigozzi, F. (2007). Performance characteristics according to playing position in elite soccer. International Journal of Sports Medicine, 28(3), 222-227. doi: 10.1055/s-2006-924294

Edwards, A. M., Macfadyen, A., \& Clark, N. (2003). Test performance indicators from a single soccer specific fitness test differentiate between highly trained and recreationally active soccer players. Journal of Sports Medicine and Physical Fitness, 43(1), 14-20.

Gorostiaga, E. M., Llodio, I., Ibáñez, J., Granados, C., Navarro, I., Ruesta, M., ... Izquierdo, M. (2009). Differences in physical fitness among indoor and outdoor elite male soccer players.
European Journal of Applied Physiology, 106(4), 483-491. doi: 10.1007/s00421-009-1040-7

Helgerud, J., Engen, L. C., Wisloff, U., \& Hoff, J. (2001). Aerobic endurance training improves soccer performance. Medicine \& Science in Sports \& Exercise, 33(11), 1925-1931.

Hoff, J., \& Helgerud, J. (2004). Endurance and strength training for soccer players: Physiological considerations. Sports Medicine, 34(3), 165180.

Impellizzeri, F. M., Rampinini, E., \& Marcora, S. M. (2005). Physiological assessment of aerobic training in soccer. Journal of Sports Sciences, 23(6), 583-592.

Komi, P. V. (2000). Stretch-shortening cycle: A powerful model to study normal and fatigued muscle. Journal of Biomechanics, 33(10), 11971206.

Markovic, G. D., Dizdar, I., Jukic, M., \& Cardinale, M. (2004). Reliability and factorial validity of squat and countermovement jump tests. Journal of Strength and Conditioning Research, 18(3), 551555.

Matos, J. A., Aidar, F. J., Mendes, R. R., Lômeu, L. M., Santos, C. A., Pains, R., ... Reis, V. M. (2008). Capacidade de aceleração de jogadores de futsal e futebol. Fitness \& Performance Journal, $7(4), 224-228$.

Reilly, T., \& Thomas, V. (1976). A motion analysis of work rate in different positional roles in professional football match-play. Journal of Human Movement Studies, 2(1), 87-97.

Seabra, A., Maia, J. A., \& Garganta, R. (2001). Crescimento, maturação, aptidão física, força explosiva e habilidades motoras específicas: Estudo em jovens futebolistas e não futebolistas do sexo masculino dos 12 aos 16 anos de idade. Revista Portuguesa de Ciências do Desporto, 1 (2), 22-35.

Silvestre, R., West, C., Maresh, C. M., \& Kraemer, W. J. (2006). Body composition and physical performance in men's soccer: A study of a national collegiate athletic association division I team. Journal of Strength and Conditioning Research, 20(1), 177-183.

Sleivert, G., \& Taingahue, M. (2004). The relationship between maximal jump-squat power and sprint acceleration in athletes. European Journal of Applied Physiology, 91 (1), 46-52. doi: 10.1007/ s00421-003-0941-0

Soares, B. H., \& Tourinho Filho, H. (2006). Análise da distância e intensidade dos deslocamentos 
numa partida de futsal nas diferentes posições de jogo. Revista Brasileira de Educação Física e Esporte, 20(2), 93-101.

Spencer, M., Bishop, D., Dawson, D., \& Goodman, C. (2005). Physiological and metabolic responses of repeated-sprint activities: Specific to field-based team sports. Sports Medicine, 35(12), 1025-1044.

Villar, R., \& Denadai, B. S. (2001). Efeitos da idade na aptidão física em meninos praticantes de futebol. Motriz, 7(2), 93-98.

Wisloff, U., Castagna, C., Helgerud, J., Jones, R., \& Hoff, J. (2004). Strong correlation of maximal squat strength with sprint performance and vertical jump height in elite soccer players. British Journal of Sports Medicine, 38 (3), 285-288.

Wisloff, U., Helgerud, J., \& Hoff, J. (1998). Strength and endurance of elite soccer players. Medicine $\&$ Science in Sports \& Exercise, 30(3), 462-667.

Wragg, C. B., Maxwell, N. S., \& Doust, J. H. (2000). Evaluation of the reliability and validity of a soccer-specific field test of repeated sprint ability. European Journal of Applied Physiology, $83(1), 77-83$.

(c) EY-Ne Todo o conteúdo da revista Motricidade está licenciado sob a Creative Commons, exceto quando especificado em contrário e nos conteúdos retirados de outras fontes bibliográficas. 